\title{
The consistency of probabilistic regresses. A reply to Jeanne Peijnenburg and David Atkinson
}

\author{
Frederik Herzberg \\ Copyright notice: The final publication, in Studia Logica 94 (2010), no. 3, pp. \\ 331-345, is available at link.springer.com, doi:10.1007/s11225-010-9242-x
}

\begin{abstract}
In a recent paper, Jeanne Peijnenburg and David Atkinson [Studia Logica, 89(3):333-341 (2008)] have challenged the foundationalist rejection of infinitism by giving an example of an infinite, yet explicitly solvable regress of probabilistic justification. So far, however, there has been no criterion for the consistency of infinite probabilistic regresses, and in particular, foundationalists might still question the consistency of the solvable regress proposed by Peijnenburg and Atkinson.

In this paper, we employ Robinsonian nonstandard analysis to prove that a probabilistic regress is already consistent if it is admissible in the sense that its forward-iteration solution does not lead to obvious contradictions; naturally, the converse also holds true. As a consequence, it turns out that there is a rich class of probabilistic regresses, which generically will fail to be solvable.

We therefore propose a weaker version of the Probabilistic Regress Problem which concedes the existence of solvable regresses, but denies their genericity.
\end{abstract}

2000 Mathematics Subject Classification. Primary: 03A05; 60A99. Secondary: $03 \mathrm{H} 05$.

Key words and phrases. probabilistic justification; regress problem; foundationalism; infinitism; nonstandard analysis.

Institut für Mathematische Wirtschaftsforschung, Universität Bielefeld, Universitätsstraße

D-33615 Bielefeld, Germany. E-mail address: fherzberg@uni-bielefeld.de. 


\section{Introduction}

This short note presents a mathematical analysis of infinite regresses of probabilistic justification (for short: probabilistic regresses) with an emphasis on their rôle in recent discussions of infinitism. A probabilistic regress is an infinite regress of epistemic justification based on a comparison of conditional probabilities at each stage of the regress. The main finding of this paper is that every probabilistic regress which satisfies certain natural conditions derived from its forward-iteration solution is mathematically consistent. At the end of this article, we discuss the implications of this result for the foundationalist rejection of infinitism.

The paper is organised as follows: We begin, in Section 2, with a review of the Probabilistic Regress Problem, largely based on the recent paper by Peijnenburg and Atkinson [7]. In Section 3 and Section 4, we formalise the notions of a probabilistic regress and its closed-form solvability; then, we briefly summarise Peijnenburg and Atkinson's 7 ] example of a probabilistic regress which allows for a closed-form solution (Section 5). In Section 6, we state and prove the main result of this paper: Every probabilistic regress whose forward-iteration solution avoids obvious contradictions already has a mathematical model (in terms of a probability space and a sequence of events on that probability space which carry the conditional probability assignments prescribed by the regress). In the final section of the paper (Section 7), we propose a modification of the foundationalist stance with respect to infinite regresses of probabilistic justification: Whilst "harmless", i.e. solvable and consistent probabilistic regresses do exist (as in the examples of Peijnenburg [6] or Peijnenburg and Atkinson [7]), probabilistic regresses are generically not solvable.

\section{The Probabilistic Regress Problem}

The Regress Problem is a long-standing foundationalist challenge to infinitism, dating back at least to the days of Russell [9] and C.I. Lewis [4]. The most basic version, henceforth called the Basic Regress Problem, reads: "If my belief in some proposition $p_{0}$ is justified by my belief in some other proposition $p_{1}$, which in turn is justified by my belief in yet another proposition $p_{2}$, and so on ad infinitum, then there is no epistemic justification for my belief in any of the propositions $p_{k}$." The probabilistic counterpart of the Basic Regress Problem, which will be called Probabilistic Regress Problem, asserts: "If my belief in some proposition $S_{0}$ is probabilistically supported by my belief in $S_{1}$, which in turn is probabilistically supported by my belief in $S_{2}$, and so on ad infinitum, then there is no probabilistic justification for my belief in any of the propositions $S_{k} . "$ (Herein, propositions are identified with events, as is ubiquitous practice in probability theory.)

There are two classical lines of argument for both the (Basic) Regress Problem and for the Probabilistic Regress Problem: Firstly, finite minds are incapable of performing an infinite number of probabilistic calculations (cf. e.g. Fumerton [3]). Secondly, inference of $S_{n}$ from $S_{n+1}$ does not originate justification for $S_{n}$, but only transfers any previous justification for $S_{n+1}$ to $S_{n}$; this means that in a regress - be it of the basic, traditional kind or of 
the probabilistic sort - none of the $S_{n}$ should actually be seen as justified (cf. e.g. Dancy [1]).

In order to clarify the assertion of the Probabilistic Regress Problem, we still need to provide a rigorous definition of the concept of probabilistic support, as introduced by Peijnenburg [6] and Peijnenburg and Atkinson [7]. Herein and in all of this paper, we shall follow the notational conventions of probability theory rather than those of propositional logic when it comes to denoting complementary or negated events; the complement of an event $A$ is denoted by $\complement A$ (rather than " $\neg A$ "). Informally speaking, an event $B$ probabilistically supports $A$ if and only if $A$ is more probable given $B$ than given non- $B$ :

Definition 1. Let $A, B$ be events on some probability space $(\Omega, \mathcal{A}, P)$.

(a) If $P(B)>0$, then the ratio $\frac{P(A \cap B)}{P(B)}$ is called the conditional probability of $A$ given $B$ and denoted $P(A \mid B)$.

(b) $B$ is contingent if and only if $0<P(B)<1$.

(c) $B$ probabilistically supports $A$ if and only if $B$ is contingent and $P(A \mid B)>P(A|\subset| C B)$.

\section{Probabilistic regresses}

Before we can analyse the Probabilistic Regress Problem formally, we need to make the notions of a probabilistic regress and of its closed-form solvability precise. This will be accomplished in Section 3 and Section 4 . respectively. In all of this paper, the set of non-negative integers will be denoted by $\mathbf{N}$.

The infinite regress outlined in the Probabilistic Regress Problem is completely determined by the probabilities $P\left(S_{k} \mid S_{k+1}\right), P\left(S_{k} \mid \complement S_{k+1}\right), k \in \mathbf{N}$ - where $P\left(S_{k} \mid S_{k+1}\right)>P\left(S_{k} \mid \complement S_{k+1}\right)$ for all $k \in \mathbf{N}$. For this reason, we identify probabilistic regresses with pairs of sequences of (conditional) probability values (i.e. elements of $[0,1]$ ) where one sequence strictly dominates the other. With this terminology, a model of a probabilistic regress is essentially a pair of a probability measure and a sequence of events which carry the conditional probability assignments specified through the regress:

Definition 2. Let $\alpha=\left\langle\alpha_{k}\right\rangle_{k \in \mathbf{N}}$ and $\beta=\left\langle\beta_{k}\right\rangle_{k \in \mathbf{N}}$ be sequences of real numbers.

(a) $\langle\alpha, \beta\rangle$ is a probabilistic regress if and only if $\alpha_{k}, \beta_{k} \in[0,1]$ and $\alpha_{k}>\beta_{k}$ for all $k \in \mathbf{N}$.

(b) Let $\langle\alpha, \beta\rangle$ be a probabilistic regress. A model of $\langle\alpha, \beta\rangle$ is a pair $\langle P, S\rangle$, wherein $P$ is a probability measure with domain $\mathcal{A}$ and $S=$ $\left\langle S_{k}\right\rangle_{k \in \mathbf{N}} \in \mathcal{A}^{\mathbf{N}}$, such that

(1)

$\forall k \in \mathbf{N} \quad 0<P\left(S_{k+1}\right)<1, \quad P\left(S_{k} \mid S_{k+1}\right)=\alpha_{k}>\beta_{k}=P\left(S_{k} \mid \boldsymbol{C} S_{k+1}\right)$.

(c) A probabilistic regress $\langle\alpha, \beta\rangle$ is consistent if there exists a model $\langle P, S\rangle$ of $\langle\alpha, \beta\rangle$.

(d) If $\langle\alpha, \beta\rangle$ is a probabilistic regress, we define

$$
\gamma_{k}=\alpha_{k}-\beta_{k}
$$

for all $k \in \mathbf{N}$. 
The analysis of probabilistic regresses would be much simpler if we were to demand that $\alpha_{k}, \beta_{k} \in(0,1)$ for all $k \in \mathbf{N}$.

REMARK 3. Whenever $\langle P, S\rangle$ is a model of some probabilistic regress $\langle\alpha, \beta\rangle$, then $P\left(S_{k}\right)>0$ for all $k \in \mathbf{N}$.

(All proofs can be found in Appendix A, )

Thus, one always has $P\left(S_{0}\right)>0$ whenever $\langle P, S\rangle$ is a model of a probabilistic regress. This refutes, in the strongest possible sense, the claim that $P\left(S_{0}\right)=0$ should hold for all models of probabilistic regresses - an assertion that can be traced back to Russell [9] and C.I. Lewis [4, p. 173] (as explained by Peijnenburg and Atkinson [7]).

\section{Solvable probabilistic regresses}

A probabilistic regress $\langle\alpha, \beta\rangle$ is solvable if for any model $\langle P, S\rangle$ of $\langle\alpha, \beta\rangle$ one can derive a closed-form expression for $P\left(S_{0}\right)$.

It is difficult to provide a precise definition of solvability, since the notion of a closed-form expression is not a well-defined mathematical concept 1 However, if there were universal agreement on the set $C$ of closed-form expressions of real numbers, a candidate for a definition of closed-form solvability would be as follows: A probabilistic regress $\langle\alpha, \beta\rangle$ is solvable (in closed form) if there exists some $p \in C \cap(0,1]$ such that for any model $\langle P, S\rangle$ of $\langle\alpha, \beta\rangle$, one can prove $P\left(S_{0}\right)=p$ (in Zermelo-Fraenkel set theory with the Axiom of Choice, say).

An important argument in favour of the Probabilistic Regress Problem is the claim that no probabilistic regress is solvable (e.g. C.I. Lewis [4]). In their recent article, Peijnenburg and Atkinson [7] have confounded this claim by providing an example of $\langle\alpha, \beta\rangle$ where one can easily derive that $P\left(S_{0}\right)=\frac{1}{2}$ for any model $\langle P, S\rangle$ of $\langle\alpha, \beta\rangle$.

In order to get a better understanding what it means, in general, for a probabilistic regress to be solvable, we note with Peijnenburg 6. Equation (13)] and Peijnenburg and Atkinson [7, Equations (4),(5)] that $P\left(S_{k}\right)$ is given by a recursion equation and disentangle this recursion equation through forward iteration:

Remark 4 (and Definition). Let $\langle P, S\rangle$ be a model of some probabilistic regress $\langle\alpha, \beta\rangle$. Then,

$$
\forall k \in \mathbf{N} \quad P\left(S_{k}\right)=\alpha_{k} P\left(S_{k+1}\right)+\beta_{k} P\left(\complement S_{k+1}\right) .
$$

\footnotetext{
${ }^{1}$ Whilst all rational numbers should certainly be counted as closed-form expressions, it is less clear, which irrational numbers should be included. Are all algebraic numbers closed-form expressions - even those which cannot be defined through radicals? What about certain transcendental numbers (like $\pi$ or the Euler number $e$ or some exogenously given constants)? Should the set of closed-form expressions be closed under the application of rational functions or special functions (e.g. exponential, trigonometric, hyperbolic functions)? All one can say is that in all of theses cases, any candidate for set of closedform expressions will be a countable set $C$ of real numbers. (The set of algebraic numbers is countably infinite, since it is the set of zeroes of polynomials with integer coefficients and there are only countably many such polynomials and they all will have only finitely many zeroes. Moreover, the set of transcendental numbers which can be regarded as closed-form expressions will be countable. Finally, the set of rational functions is countable and the set of special functions is also countable.)
} 
$S O$

$$
\forall k \in \mathbf{N} \quad P\left(S_{k}\right)=\beta_{k}+\gamma_{k} P\left(S_{k+1}\right),
$$

hence by forward iteration

(4) $\quad \forall k, n \in \mathbf{N} \quad P\left(S_{k}\right)=P\left(S_{k+n+1}\right) \prod_{i=0}^{n} \gamma_{i+k}+\sum_{\ell=0}^{n} \beta_{\ell+k} \prod_{i=0}^{\ell-1} \gamma_{i+k}$

and finally

(5) $\quad \forall k \in \mathbf{N} \quad P\left(S_{k}\right)=\lim _{n \rightarrow \infty} P\left(S_{k+n+1}\right) \prod_{i=0}^{n} \gamma_{i+k}+\sum_{\ell=0}^{\infty} \beta_{\ell+k} \prod_{i=0}^{\ell-1} \gamma_{i+k}$,

provided the limits exist.

For $k=0$, we obtain the equation

$$
\forall n \in \mathbf{N} \quad P\left(S_{0}\right)=P\left(S_{n+1}\right) \prod_{i=0}^{n} \gamma_{i}+\sum_{\ell=0}^{n} \beta_{\ell} \prod_{i=0}^{\ell-1} \gamma_{i},
$$

which Peijnenburg and Atkinson [7, Equations (6)] already mention. In particular,

$$
P\left(S_{0}\right)=\lim _{n \rightarrow \infty} P\left(S_{n+1}\right) \prod_{i=0}^{n} \gamma_{i}+\sum_{\ell=0}^{\infty} \beta_{\ell} \prod_{i=0}^{\ell-1} \gamma_{i}
$$

provided the limits exist.

\section{An example of a solvable probabilistic regress}

Peijnenburg [6 and Peijnenburg and Atkinson 7] have suggested examples of probabilistic regresses in which $P\left(S_{0}\right)$ can be easily computed via Equation (7), thus resolving the regress and confounding the two aforementioned foundationalist objections - the finite-mind and the transfer objection - for these particular examples of regresses.

Peijnenburg and Atkinson [7] studied the sequences $\alpha, \beta$ defined by

$$
\forall k \in \mathbf{N} \quad \alpha_{k}=1-\frac{1}{k+3}, \quad \beta_{k}=\frac{1}{k+3},
$$

hence

$$
\forall k \in \mathbf{N} \quad \gamma_{k}=\frac{k+1}{k+3}
$$


For these choices of $\alpha$ and $\beta$, calculations ${ }^{2}$ lead to (9) $P\left(S_{0}\right)=\lim _{n \rightarrow \infty} P\left(S_{n+1}\right) \frac{2}{(n+2)(n+3)}+\frac{1}{3}+\frac{1}{2}-\frac{1}{3}-\lim _{n \rightarrow \infty}\left(\frac{1}{n+2}-\frac{1}{n+3}\right)$, thus

$$
P\left(S_{0}\right)=\frac{1}{2}
$$

Hence, this particular probabilistic regress is easily solvable, refuting the foundationalist view of regresses in principle. Following Peijnenburg [6] we note that a model of the probabilistic regress $\langle\alpha, \beta\rangle$ is solvable if both (i) $\sum_{k=0}^{n} \beta_{k} \prod_{i=0}^{k-1}\left(\alpha_{i}-\beta_{i}\right)$, which is the second term in Equation (6), can be explicitly calculated (at least in the limit as $n \rightarrow \infty$ ) with a closed-form solution and (ii) there exists some constant $c_{0}$ such that

$$
\forall k \in \mathbf{N} \quad 1>c_{0}>\alpha_{k}-\beta_{k}>0 .
$$

For instance, this is the case if $\alpha$ and $\beta$ are just constant and $\alpha_{1}>\beta_{1}$.

The question remains, however, whether probabilistic regresses based on certain sequences $\alpha, \beta$ - in particular, for the sequences suggested by Peijnenburg [6] and Peijnenburg and Atkinson [7] - actually exist: Given sequences $\alpha, \beta$ satisfying some obvious conditions which follow from Equation (5) and the definition of a probabilistic regress, is there a (countably additive) probability measure $P$ and a sequence of events $S$ such that $\langle P, S\rangle$ is a model of the probabilistic regress $\langle\alpha, \beta\rangle$ ? The answer is affirmative, as we shall see in the next section.

\section{The consistency of probabilistic regresses}

REMARK 5. Let $\langle\alpha, \beta\rangle$ be a probabilistic regress. Then, the limit $\prod_{k=0}^{\infty} \gamma_{k}$ exists (and is $\geq 0$ ).

We now introduce a necessary criterion for the consistency of a probabilistic regress, viz. admissibility. Informally speaking, a probabilistic regress is admissible if and only if Equation (5) does not immediately lead to contradictions. Interestingly, it will turn out that admissibility of a probabilistic regress is also sufficient for consistency.

Definition 6. A probabilistic regress $\langle\alpha, \beta\rangle$ is admissible if either

$$
\text { - } \prod_{k=0}^{\infty} \gamma_{k}>0 \text {, or }
$$

$$
\begin{aligned}
& \forall n \in \mathbf{N} \quad \prod_{i=0}^{n} \gamma_{i}=\frac{2}{(n+2)(n+3)} \\
& \forall k \in \mathbf{N} \quad \beta_{k} \prod_{i=0}^{k-1} \gamma_{i}=\frac{1}{k+3} \frac{2}{(k+1)(k+2)}=\frac{1}{k+1}-\frac{2}{k+2}+\frac{1}{k+3} \\
& =\frac{1}{k+1}-\frac{1}{k+2}-\left(\frac{1}{k+2}-\frac{1}{k+3}\right),
\end{aligned}
$$

whence the second term in Equation (6) is a telescoping sum. Summarising this, one obtains Equation (9). 
- (a) $\beta_{k}>0$ for infinitely many $k$, and

(b) $\sum_{\ell=0}^{\infty} \beta_{\ell+k} \prod_{i=0}^{\ell-1} \gamma_{i+k}<1$ for all $k \in \mathbf{N}$.

Indeed, if $\langle\alpha, \beta\rangle$ is not admissible, then Equation (5) readily rules out the existence of a model of $\langle\alpha, \beta\rangle$, as one can see from the proof of the following Lemma:

Lemma 7. Let $\langle\alpha, \beta\rangle$ be a probabilistic regress. If $\langle\alpha, \beta\rangle$ is not admissible, then it is not consistent.

So, admissibility of a probabilistic regress is a necessary condition for consistency. The main theorem of this paper shows that the converse is also true.

Theorem 8 (Consistency of Probabilistic Regresses). A probabilistic regress $\langle\alpha, \beta\rangle$ is consistent if and only if it is admissible.

The proof of the sufficiency of admissibility for consistency is obtained by taking the nonstandard limit (as $n \rightarrow \infty$ ), in the sense of Robinsonian nonstandard analysis [8] and Loeb probability theory [5], of the following lemma:

Lemma 9. Let $\alpha, \beta \in[0,1]^{n}$, let $\varepsilon_{n} \in(0,1)$ and suppose $\alpha_{k}>\beta_{k}$ for all $k \leq n$. Then there exist a probability space $(\Omega, \mathcal{A}, P)$ as well as events $S_{0}^{n, \varepsilon_{n}}, \ldots, S_{n}^{n, \varepsilon_{n}} \in \mathcal{A}$ such that $0<P\left(S_{k}^{n, \varepsilon_{n}}\right)<1$ for all $k \leq n$ and

(10) $\quad \forall k<n-1 \quad P\left(S_{k}^{n, \varepsilon_{n}} \mid S_{k+1}^{n, \varepsilon_{n}}\right)=\alpha_{k}>\beta_{k}=P\left(S_{k}^{n, \varepsilon_{n}} \mid \complement S_{k+1}^{n, \varepsilon_{n}}\right)$

as well as $P\left(S_{n}^{n, \varepsilon_{n}}\right)=\varepsilon_{n}$.

Theorem 8 can be used to verify that the solvable probabilistic regress proposed by Peijnenburg and Atkinson [7] (see Section 5 above) is admissible and hence consistent. 3

From Theorem 8, we may also deduce that any conceivable value $p$ of $P\left(S_{0}\right)$ is attained in a model of some probabilistic regress:

COROllary 10. For every $p \in(0,1)$, there exists some probabilistic regress $\langle\alpha, \beta\rangle$, such that $P\left(S_{0}\right)=p$ holds for any model $\langle P, S\rangle$ of $\langle\alpha, \beta\rangle$.

$$
\begin{aligned}
& \text { 3. In order to prove that the regress defined in Section } 5 \text { is admissible, first remark that } \\
& \beta_{k}>0 \text { holds for all } k \in \mathbf{N} \text {. Consider an arbitrary } k \in \mathbf{N} \text {. Then, using Equation } 8 \text {, } \\
& \qquad \begin{aligned}
\sum_{\ell=0}^{\infty} \beta_{\ell+k} \prod_{i=0}^{\ell-1} \gamma_{i+k} & =\sum_{\ell=0}^{\infty} \beta_{\ell+k} \prod_{i=k}^{\ell+k-1} \gamma_{i}=\sum_{\ell=k}^{\infty} \beta_{\ell} \prod_{i=k}^{\ell-1} \gamma_{i} \\
& =\sum_{\ell=k}^{\infty} \beta_{\ell} \prod_{i=0}^{\ell-1} \gamma_{i} / \prod_{j=0}^{k-1} \gamma_{j}=\sum_{\ell=k}^{\infty} \beta_{\ell} \prod_{i=0}^{\ell-1} \gamma_{i} / \prod_{j=0}^{k-1} \gamma_{j} \\
& =\sum_{\ell=k}^{\infty} \frac{1}{\ell+3} \frac{2}{(\ell+1)(\ell+2)} \frac{(k+1)(k+2)}{2} \\
& =\left(k^{2}+3 k+2\right) \sum_{\ell=k}^{\infty} \frac{1}{(\ell+1)(\ell+2)(\ell+3)}
\end{aligned}
\end{aligned}
$$

Note that

$$
\begin{aligned}
\forall \ell \in \mathbf{N} \quad(\ell+1)(\ell+2)(\ell+3) & =(\ell+1)\left(\ell^{2}+5 \ell+6\right)=\ell^{3}+6 \ell^{2}+11 \ell+6 \\
& \geq \ell^{3}+\frac{27}{5} \ell^{2}+\frac{243}{25} \ell+\frac{729}{125}=(\ell+9 / 5)^{3}
\end{aligned}
$$


In all of this paper, we have required $P$ to be countably additive - so that standard terminology from probability theory could be used. However, at least for questions of consistency and admissibility, this requirement is redundant: Suppose that $P$ is a finitely additive probability measure on an algebra $\mathcal{A}$, and let $S=\left\langle S_{k}\right\rangle_{k \in \mathbf{N}}$ be a sequence of algebra elements that satisfies Equation (1) for some probabilistic regress $\langle\alpha, \beta\rangle$. Then, one can still derive Equation (5). Hence, if $\langle\alpha, \beta\rangle$ were not admissible, we could mimick the proof of Lemma 7 to produce a contradiction. For this reason, any regress which is not admissible will not even have a finitely additive model. Theorem 8 can thus be strengthened as follows: A probabilistic regress is admissible if and only if it has a finitely additive model, in which case it even has a (countably additive) model. Hence, in the analysis of probabilistic regresses, the distinction between finitely additive and countably additive probability measures turns out to be irrelevant. (For a systematic comparison of finitely additive and countably additive probability measures in epistemic contexts, cf. e.g. Schurz and Leitgeb [10].)

\section{The nongenericity of solvable probabilistic regresses}

The main theorem of this article establishes that every admissible probabilistic regress $\langle\alpha, \beta\rangle$ possesses a model, i.e. there exists a probability

whence

$$
\begin{aligned}
\sum_{\ell=0}^{\infty} \beta_{\ell+k} \prod_{i=0}^{\ell-1} \gamma_{i+k} & \leq\left(k^{2}+3 k+2\right) \sum_{\ell=k}^{\infty} \frac{1}{(\ell+9 / 5)^{3}} \\
& \leq\left(k^{2}+3 k+2\right) \sum_{\ell=k}^{\infty} \int_{\ell-1}^{\ell}(x+9 / 5)^{-3} \mathrm{~d} x \\
& =\left(k^{2}+3 k+2\right) \int_{k-1}^{\infty}(x+9 / 5)^{-3} \mathrm{~d} x \\
& =\left(k^{2}+3 k+2\right)\left[-\frac{(x+9 / 5)^{-2}}{2}\right]_{x=k-1}^{\infty} \\
& =\left(k^{2}+3 k+2\right) \frac{(k+4 / 5)^{-2}}{2}=\frac{1}{2} \frac{k^{2}+3 k+2}{k^{2}+8 k / 5+16 / 25} \\
& =\frac{k^{2}+3 k+2}{2 k^{2}+16 k / 5+32 / 25}
\end{aligned}
$$

Now,

$$
\frac{k^{2}+3 k+2}{2 k^{2}+16 k / 5+32 / 25} \geq 1 \Leftrightarrow k^{2}+k / 5-18 / 25 \leq 0 \Leftrightarrow(k+0.1)^{2} \leq 0.73,
$$

SO

$$
\forall k \in \mathbf{N}_{>0} \quad \frac{k^{2}+3 k+2}{2 k^{2}+16 k / 5+32 / 25}<1
$$

and hence by the previous chain of inequalities $\sum_{\ell=0}^{\infty} \beta_{\ell+k} \prod_{i=0}^{\ell-1} \gamma_{i+k}<1$ for all $k \in \mathbf{N}_{>0}$. For $k=0$, Equation 11 yields

$$
\begin{aligned}
\sum_{\ell=0}^{\infty} \beta_{\ell} \prod_{i=0}^{\ell-1} \gamma_{i} & =2 \sum_{\ell=0}^{\infty} \frac{1}{(\ell+1)(\ell+2)(\ell+3)} \\
& =2 \sum_{\ell=1}^{\infty} \frac{1}{\ell(\ell+1)(\ell+2)}=2 \times \frac{1}{4}=\frac{1}{2}<1
\end{aligned}
$$

therefore $\sum_{\ell=0}^{\infty} \beta_{\ell+k} \prod_{i=0}^{\ell-1} \gamma_{i+k}<1$ holds for all $k \in \mathbf{N}$. Hence, this particular regress is admissible and thus consistent. 
measure $P$ and a sequence $S$ of $P$-measurable events which satisfy the conditional probability assignments given by $\langle\alpha, \beta\rangle$ through Equation (1). A corollary of that theorem shows that $P\left(S_{0}\right)$ can attain just any value between 0 and 1 (exclusive) in some probabilistic regress. It follows that there exist solvable consistent probabilistic regresses. In another application of the theorem (footnote 3), we have verified the consistency of the simple solvable regress that was recently constructed by Peijnenburg and Atkinson [7.

An inspection of the examples of Peijnenburg [6] and Peijnenburg and Atkinson [7] immediately reveals, however, that the solvability of these regresses depends on some kind of gerrymandering with $\alpha$ and $\beta$, in order to obtain (i) certain combinatorial features which lead to cancellations in the second term of Equation (7) and (ii) certain topological peculiarities which ensure that the first term in Equation (7) vanishes. This, however, is definitely not a generic feature of probabilistic regresses.

Therefore, even after the findings of Peijnenburg and Atkinson [7] and of this article, the foundationalists might still argue as follows: Notwithstanding the fact that there are solvable consistent regresses, at least generically, a probabilistic regress does not admit a closed-form solution, let alone a solution that can be verified with a short proof (as in the example of Peijnenburg and Atkinson [7]). This suffices to support a new, although admittedly rather weak version of the (Probabilistic) Regress Problem: "If my belief in some proposition $S_{0}$ is probabilistically supported by my belief in $S_{1}$, which in turn is probabilistically supported by my belief in $S_{2}$, and so on ad infinitum, then there might be a probabilistic justification for my belief in proposition $S_{0}$, but generically there is no closed-form solution for $P\left(S_{0}\right)$." The probability $P\left(S_{0}\right)$ will be strictly positive for any probabilistic regress (see Remark 3), but generically we are unable to calculate or estimate it explicitly; for a given regress, $P\left(S_{0}\right)$ might be very close to zero.

\section{Appendix A. Proofs}

Proof of Remark 3. We only have to show that $P\left(S_{0}\right)>0$. However, from the inequalities (1), one gets $P\left(S_{0} \mid S_{1}\right)=\alpha_{0}>0$, which means that $P\left(S_{1}\right)>0$ and $P\left(S_{0} \cap S_{1}\right)>0$, thus $P\left(S_{0}\right)>0$.

Proof of Remark 4, Let $k \in \mathbf{N}$. Then, by the definition of a probabilistic regress, we have

$$
P\left(S_{k}\right)=P\left(S_{k} \cap S_{k+1}\right)+P\left(S_{k} \cap \complement S_{k+1}\right)=\alpha_{k} P\left(S_{k+1}\right)+\beta_{k} P\left(\complement S_{k+1}\right),
$$

which is Equation (2). Equation (2) immediately yields Equation (3). From here, mathematical induction in $n$ leads to

$$
\forall k, n \in \mathbf{N} \quad P\left(S_{k}\right)=P\left(S_{k+n+1}\right) \prod_{i=0}^{n} \gamma_{i+k}+\sum_{\ell=0}^{n} \beta_{\ell+k} \prod_{i=0}^{\ell-1} \gamma_{i+k}
$$

(where we use the convention $\prod_{i=0}^{-1} \gamma_{i}=1$ ). In the limit, one obtains Equation (5), provided the limits on the right-hand side of Equation (5) exist. 
Proof of Remark 5. For all $n \in \mathbf{N}$, one has $\prod_{k=0}^{n} \gamma_{k} \geq 0$. On the other hand, $0 \leq \beta_{k}<\alpha_{k} \leq 1$ means that $0<\gamma_{k} \leq 1$, whence $\prod_{k=0}^{n+1} \gamma_{k} \leq$ $\prod_{k=0}^{n} \gamma_{k}$ for all $n \in \mathbf{N}$. Therefore, $\left\langle\prod_{k=0}^{n} \gamma_{k}\right\rangle_{n \in \mathbf{N}}$ is a decreasing sequence which is bounded from below (by 0), hence it must have a (nonnegative) limit, viz. $\inf \left\{\prod_{k=0}^{n} \gamma_{k}: n \in \mathbf{N}\right\}$.

Proof of Lemma 7, Suppose $\langle\alpha, \beta\rangle$ is not admissible. There are two cases to be considered:

(a) $\prod_{k=0}^{\infty}\left(\alpha_{k}-\beta_{k}\right)=0$ and $\beta_{k}=0$ for all but finitely many $k$. In this case, there is some $m \in \mathbf{N}$ such that $\beta_{k}=0$ for all $k \geq m$, and Equation (5) already yields that $P\left(S_{k}\right)=0$ for all $k \geq m$. This contradicts the definition of a probabilistic regress.

(b) $\prod_{k=0}^{\infty}\left(\alpha_{k}-\beta_{k}\right)=0$ and $\sum_{\ell=0}^{\infty} \beta_{\ell+k} \prod_{i=0}^{\ell-1}\left(\alpha_{i+k}-\beta_{i+k}\right) \geq 1$ for some $k \in \mathbf{N}$. Then $P\left(S_{k}\right) \geq 1$ due to Equation (5), so $P\left(S_{k}\right)=1$, which again contradicts the definition of a probabilistic regress.

First, we will give the proof of Theorem 8 afterward, we shall turn to the proof of Lemma 9.

Proof of Theorem 8, Lemma 7 shows that consistency of a probabilistic regress implies admissibility.

We will now prove the converse. Let $\langle\alpha, \beta\rangle$ be a probabilistic regress. In the notation of Lemma 9, consider the probability space $\left({ }^{*} \Omega,{ }^{*} \mathcal{A},{ }^{*} P\right)$ and the double-indexed family ${ }^{*}\left\langle S_{k}^{n}: k \leq n, \quad n \in \mathbf{N}\right\rangle=$ $\left\langle{ }^{*} S_{k}^{n, \varepsilon_{n}}: k \leq n, \quad n \in{ }^{*} \mathbf{N}\right\rangle$. The Loeb probability space $\left({ }^{*} \Omega, \sigma\left({ }^{*} \mathcal{A}\right), \mathrm{L}\left({ }^{*} P\right)\right)$ (cf. Loeb [5]) will eventually furnish us with a model of the probabilistic regress $\langle\alpha, \beta\rangle$.

By the Transfer Principle of Robinsonian nonstandard analysis [8], we must have $0<{ }^{*} P\left(S_{k}^{n, \varepsilon_{n}}\right)<1$ for all $n \in{ }^{*} \mathbf{N}$ and $k \leq n$ as well as for all $n \in{ }^{*} \mathbf{N}$ and $k<n$

$$
{ }^{*} P\left({ }^{*} S_{k}^{n, \varepsilon_{n}} \mid{ }^{*} S_{k+1}^{n, \varepsilon_{n}}\right)={ }^{*} \alpha_{k}>{ }^{*} \beta_{k}={ }^{*} P\left({ }^{*} S_{k}^{n, \varepsilon_{n}} \mid \mathbb{C}^{*} S_{k+1}^{n, \varepsilon_{n}}\right) .
$$

Therefore, if we fix any $c \in(0,1)$, we have for any infinite $N \in{ }^{*} \mathbf{N}$ and every $k \in \mathbf{N}$ that $0<{ }^{*} P\left({ }^{*} S_{k+1}^{N, c}\right)<1$ and

$$
{ }^{*} P\left({ }^{*} S_{k}^{N, c} \mid{ }^{*} S_{k+1}^{N, c}\right)=\alpha_{k}>\beta_{k}={ }^{*} P\left({ }^{*} S_{k}^{N, c} \mid \complement^{*} S_{k+1}^{N, c}\right) .
$$

This implies

$$
{ }^{*} P\left({ }^{*} S_{k}^{N, c}\right)=\beta_{k}+\left(\alpha_{k}-\beta_{k}\right){ }^{*} P\left({ }^{*} S_{k+1}^{N, c}\right),
$$

and by iteration, we obtain for all $k, n \in \mathbf{N}$,

$$
{ }^{*} P\left({ }^{*} S_{k}^{N, c}\right)={ }^{*} P\left({ }^{*} S_{k+n}^{N, c}\right) \prod_{i=0}^{n} \gamma_{k+i}+\sum_{\ell=0}^{n} \beta_{k+\ell} \prod_{i=0}^{\ell-1} \gamma_{k+i}
$$

(analogously to Equation (4)), hence in particular

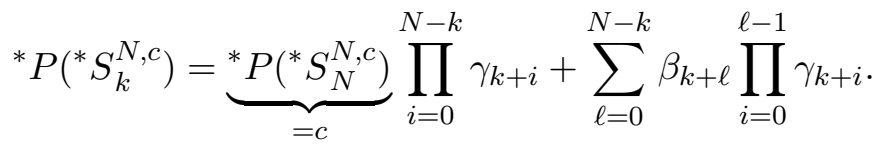


If $\prod_{i=0}^{\infty} \gamma_{i}>0$, then for every infinite $N \in{ }^{*} \mathbf{N}$, one has $\prod_{i=0}^{N} \gamma_{i} \gg 0$. If $\beta_{\ell}>0$ for infinitely many $\ell$, then there must be some $\beta_{\ell}>0$ with $k \leq \ell \in \mathbf{N}$. In both cases, Equation (13) will yield that ${ }^{*} P\left({ }^{*} S_{k}^{N, c}\right) \gg 0$ (for arbitrary $k \in \mathbf{N})$. Moreover, Equation (13) shows that ${ }^{*} P\left({ }^{*} S_{k}^{N, c}\right) \ll{ }^{*} P\left({ }^{*} S_{k}^{N, c^{\prime}}\right)$ for any $c^{\prime} \in(c, 1)$, hence ${ }^{*} P\left({ }^{*} S_{k}^{N, c}\right) \ll 1$.

Finally, we obtain from Equation (12) that

$$
\mathrm{L}\left({ }^{*} P\right)\left({ }^{*} S_{k}^{N, c} \mid{ }^{*} S_{k+1}^{N, c}\right)=\alpha_{k}>\beta_{k}=\mathrm{L}\left({ }^{*} P\right)\left({ }^{*} S_{k}^{N, c} \mid \complement^{*} S_{k+1}^{N, c}\right)
$$

for all $k \in \mathbf{N}$.

Thus, $\left\langle\mathrm{L}\left({ }^{*} P\right),\left\langle{ }^{*} S_{k}^{N, c}\right\rangle_{k \in \mathbf{N}}\right\rangle$ is a model of the probabilistic regress $\langle\alpha, \beta\rangle$.

Now, let us turn to the proof of Lemma 9. The probability space $(\Omega, \mathcal{A}, P)$ whose existence is asserted in the Lemma will be an arbitrary atomless probability space; for the sake of completeness, let us recall the definition.

Definition 11. A probability space $(\Omega, \mathcal{A}, P)$ is called atomless if and only if for all $A \in \mathcal{A}$ with $P(A)>0$, there exists some $B \subset A$ such that $0<P(B)<P(A)$.

For example, the probability space given by the Lebesgue measure on the unit interval $[0,1]$ is atomless.

The proof of Lemma 9 uses the following equivalent characterisation of atomlessness:

Proposition 12. A probability space $(\Omega, \mathcal{A}, P)$ is atomless if and only if for all $A \in \mathcal{A}$ and for every $\alpha \in[0,1]$ there exists some $B \subseteq A$ such that $P(B)=\alpha P(A)$.

Proof of Proposition 12. See e.g. Fremlin's treatise [2, p. 46, 215D].

Proof of Lemma 9, Let $(\Omega, \mathcal{A}, P)$ be an atomless probability space. The sets $S_{k}^{n, \varepsilon_{n}}$ will be constructed through backward recursion in $k$. For the initial step of the recursion note that since $(\Omega, \mathcal{A}, P)$ is atomless, Proposition 12 yields the existence of some event $S_{n}^{n, \varepsilon_{n}}$ with probability $\varepsilon_{n}$.

Now suppose $S_{k+1}^{n, \varepsilon_{n}}, \ldots, S_{n}^{n, \varepsilon_{n}}$ with

$$
\forall \ell>k \quad P\left(S_{\ell}^{n, \varepsilon_{n}}\right)>0
$$

and

$$
\forall \ell \in\{k+1, \cdots, n-1\} \quad P\left(S_{\ell}^{n, \varepsilon_{n}} \mid S_{\ell+1}^{n, \varepsilon_{n}}\right)=\alpha_{\ell}>\beta_{\ell}=P\left(S_{\ell}^{n, \varepsilon_{n}} \mid \complement S_{\ell+1}^{n, \varepsilon_{n}}\right)
$$

are given. We have to construct $S_{k}^{n, \varepsilon_{n}}$. Since $(\Omega, \mathcal{A}, P)$ is atomless, Proposition 12 teaches that there must be some subset $A_{k}^{n}$ of $S_{k+1}^{n, \varepsilon_{n}}$ of probability $\alpha_{k} P\left(S_{k+1}^{n, \varepsilon_{n}}\right)$ and that there must also be a subset $B_{k}^{n}$ of $\complement S_{k+1}^{n, \varepsilon_{n}}$ of probability $\beta_{k} P\left(\complement S_{k+1}^{n, \varepsilon_{n}}\right)$. Finally, we put

$$
S_{k}^{n, \varepsilon_{n}}=A_{k}^{n} \cup B_{k}^{n}
$$

Then, since $\alpha_{k}>\beta_{k} \geq 0$ and $P\left(S_{k+1}^{n, \varepsilon_{n}}\right)>0$, we readily have

$$
P\left(S_{k}^{n, \varepsilon_{n}}\right) \geq P\left(A_{k}^{n}\right)=\alpha_{k} P\left(S_{k+1}^{n, \varepsilon_{n}}\right)>0 .
$$


Moreover, we see that

$$
\alpha_{k}=\frac{P\left(A_{k}^{n}\right)}{P\left(S_{k+1}^{n, \varepsilon_{n}}\right)}=\frac{P\left(S_{k}^{n, \varepsilon_{n}} \cap S_{k+1}^{n, \varepsilon_{n}}\right)}{P\left(S_{k+1}^{n, \varepsilon_{n}}\right)}=P\left(S_{k}^{n, \varepsilon_{n}} \mid S_{k+1}^{n, \varepsilon_{n}}\right)
$$

$\left(\right.$ as $\left.P\left(S_{k+1}^{n, \varepsilon_{n}}\right)>0\right)$ and

$$
\beta_{k}=\frac{P\left(B_{k}^{n}\right)}{P\left(\complement S_{k+1}^{n, \varepsilon_{n}}\right)}=\frac{P\left(S_{k}^{n, \varepsilon_{n}} \cap \complement S_{k+1}^{n, \varepsilon_{n}}\right)}{P\left(\complement S_{k+1}^{n, \varepsilon_{n}}\right)}=P\left(S_{k}^{n, \varepsilon_{n}} \mid \complement S_{k+1}^{n, \varepsilon_{n}}\right)
$$

as $P\left(\complement S_{k+1}^{n, \varepsilon_{n}}\right)>0$.

Proof of Corollary 10. Let $p \in(0,1)$, and put $q=\frac{2 p}{p+1}$. Define $\alpha_{k}=q$ and $\beta_{k}=\frac{q}{2}$ for all $k \in \mathbf{N}$. Then, $\gamma_{i}=\frac{q}{2}$ for all $i \in \mathbf{N}$. Then, for every $k \in \mathbf{N}$, one has both $\alpha_{k}>\beta_{k}>0$ and

$$
\begin{aligned}
\sum_{\ell=0}^{\infty} \beta_{\ell+k} \prod_{i=0}^{\ell-1} \gamma_{i+k} & =\frac{q}{2} \sum_{\ell=0}^{\infty}\left(\frac{q}{2}\right)^{\ell}=\frac{q}{2} \frac{1}{1-\frac{q}{2}}=\frac{q}{2-q} \\
& =p .
\end{aligned}
$$

Therefore, $\langle\alpha, \beta\rangle$ is an admissible probabilistic regress, whence, by Theorem 8 , it must have a model $\langle P, S\rangle$. Finally, we have

$$
\lim _{n \rightarrow \infty} P\left(S_{n+1}\right) \prod_{i=0}^{n} \gamma_{i}=\lim _{n \rightarrow \infty} P\left(S_{n+1}\right)\left(\frac{q}{2}\right)^{n+1}=0
$$

by sandwiching, and if we insert $k=0$ in Equation (14), we have $\sum_{\ell=0}^{\infty} \beta_{\ell} \prod_{i=0}^{\ell-1} \gamma_{i}=p$. Therefore, Equation (7) readily shows that $P\left(S_{0}\right)=$ $p$.

\section{References}

[1] J. Dancy. Introduction to contemporary epistemology. Oxford, UK: Blackwell, 1982.

[2] D.H. Fremlin. Measure theory. Vol. 2. Colchester, UK: Torres Fremlin, 2003.

[3] R. Fumerton. Epistemology. Oxford, UK: Blackwell, 2006.

[4] C.I. Lewis. The given element in empirical knowledge. Philosophical Review, 61:168172,1952

[5] P.A. Loeb. Conversion from nonstandard to standard measure spaces and applications in probability theory. Transactions of the American Mathematical Society, 211:113122, 1975.

[6] J. Peijnenburg. Infinitism regained. Mind, 116(463):597-602, 2007.

[7] J. Peijnenburg and D. Atkinson. Probabilistic justification and the regress problem. Studia Logica, 89(3):333-341, 2008.

[8] A. Robinson. Non-standard analysis. Studies in Logic and the Foundations of Mathematics. Amsterdam: North-Holland, 1966.

[9] B. Russell. Human knowledge. London, UK: George Allen and Unwin, 1948.

[10] G. Schurz and H. Leitgeb. Finitistic and frequentistic approximation of probability measures with or without $\sigma$-additivity. Studia Logica, 89(2):257-283, 2008. 